\title{
Deep Learning Method and Infrared Imaging as a Tool for Transformer Faults Detection
}

\author{
Dragan Mlakić ${ }^{1}$, Srete Nikolovski ${ }^{2}$ and Ljubomir Majdandžic ${ }^{3}$ \\ 1. Distribution Company, Electric Power Company HZ-HB Inc. Mostar, Vilenička bb 72295, Novi Travnik, Bosnia and Herzegovina \\ 2. Computer Science and Information Technology, Faculty of Electrical Engineering, J. J. Strossmayer University, K. Trpimira 2 B, \\ Osijek, Croatia \\ 3. Environmental Protection and Energy Efficiency Fund Radnička cesta 80, Zagreb 10000, Croatia
}

\begin{abstract}
In behaviour recognition, the development of the DL (Deep Learning) method introduced massive improvements in the field of artificial intelligence, where DL represents an upgrade of the present ANN (artificial neural network) architecture. Deep Learning as a comprehensive new field of artificial intelligence completely covers the neural networks architecture that is devised to carry out certain forms of identification, such as behaviour, forms of things, trends, similarities in complex forms, etc. Regarding thermography in energy, the cases used to illustrate this are photographs of active energy components in the plant. Failures that are seen with thermography cannot be recognized by other methods. However, an expert needs to do segmentation of focusing and classification of failures. The need for daily sampling and expert work is growing. With the DL method, it can be done in real time any time. One of the popular network architectures for using DL in image analysis is the recognition algorithm - CNN (convolution neural network). Traditional artificial intelligence methods require determining factors and computations, leading to training algorithm. Machine learning has important features as well as the right weight to make decisions about new input data. This work presents DL as a flexible and adaptive method for the analysis of thermal images of energy facilities, as well as a tool used for the construction and implementation of an efficient fault analysis on the $10 / 0.4 \mathrm{kV}$ service transformer.
\end{abstract}

Key words: DL, electric components, transformers, infrared imaging, photograph analysis, CNN.

\section{Introduction}

The DL (Deep Learning) method has been significantly enhanced as a technique for improving primary artificial intelligence (AI) recognition tasks, such as object discovery, speech and image recognition, machine and human behaviour, etc. The essence of DL architecture is to allow access to solutions to more complex problems which are by nature in field of AI [1]. As a follow-up, many experts have expanded the DL's scope to many everyday problems we face, such as: face, speech, and motion detection. Research of DL is a new trend in AI science but many scientists have already done much work on explaining and developing a more detailed self-learning mechanism

Corresponding author: Srete Nikolovski, Ph.D., professor, research fields: power system analysis, power system modeling, power system protection, power system reliability. for DL [2].

With the help of many known methods for neural networks (i.e. Back Propagation), DL solves the above-mentioned everyday issues on the spot, like image classification or shape recognition [3]. Some scientists used NN (neuron networks) in their studies and they had success in modelling the method for dealing with the mentioned issues [5-7]. Many articles are based on pure neural network application to recognize objects in images based on the human visual cortex. However, there is no practical example of usage in situations like real technical problems such as protection systems, online fault recognition, power curtailment based on heat transfer. This paper presents situation where one of Deep Learning method is suitable for usage in power transformer fault recognition. Other studies are conducted focusing on 
harmonic detection with thermal recording [9], oil aging of high voltage transformer with thermal analysis [10], and this paper presents new approach to IR image analysis with the same goal-damage prevention.

For the purpose of this research, only one of the DL-modification architectures is used, which is primarily designed to identify faces in images, and which is called CNN (convolution neural network) [4]. The tool specifically designed for this type of action is AlexNet, an add-on to the Matlab software suite, which allows the entry of manually built structures into its user request framework. The first part is concerned with the subject of power transformer from the distribution network in the field of security and efficiency of operation of the distribution network as a whole. Essentially, thermographic images of transformers are analysed with a focus on the degree of intensity of heat energy and its distribution. In the second part, $\mathrm{CNN}$ is considered as a tool for basic analysis of the issues mentioned in the 1st section, and it explains a set of rules adjusted to the thermographic image of the transformer. The IR image is transferred, using Matlab, to the tool area and the results of the analysis are the output that we adjust to the desired result. Chapter 3 shows a concise example of a CNN application in the IR camera image. In case study IR images are analysed through $\mathrm{CNN}$ application and output results show focus of temperature. Focus is considered, based on image angle, like fault on energy transformer or not. Thermography expert knowledge is needed for further operations.

\section{Electromagnetic Components of Thermal Analysis}

Prematurely disconnected power supply in the distribution network almost always results in a loss of the energy supply, increased costs of managing and maintaining costs, unsatisfied customers and the ultimate loss of economic gain. According to ASNT (the American Society for Non-destructive Testing), failure of electrical equipment accounts for about $30 \%$ of the cost, as established by primary and engineering companies after a loss analysis [8].

The method for failure diagnosis is contactless, meaning it is suitable for transformer stations, and measurements were obtained using a thermal camera (Infra-Red). As a diagnostic technology, IR thermography gives immediate insight and corroborates thermal capabilities by identifying disorder, and it enables the user to combine them in a precise and clear manner, without physical contact. Following the measurement, the user obtains an actual digital document; this document is presentation of the thematic object, which can be incorporated in the final report if required. With the aim of better presentations, this paper is limited to distribution transformers ratings $10 / 0.4 \mathrm{kV}$ and commutation equipment, but the diagnostic method can be applied to all transformers in terms of physical properties of materials.

Any transformer first heats up before it burns. Heating of the transformer is the first indication of malfunction. Two types of energy transformers are used: dry and oiled. Both types provide power supply as needed: single-phase or three-phase. The porcelain mask covering the transformer conductors can hide serious faults that can be present for years without being detected. A hot spot close to the top of the conductor is usually the result of a bad conductor connection. When there are internal problems with the transformer, the focal points are usually located at the bottom of the conductor. Any doubts can be easily verified with the resistance test of individual conductors on the transformer. If the entire bus field is equal to heat distribution, or has a focal point somewhere else, not at the top and bottom, the bus field is probably in a poor condition, which is confirmed by the inductance test.

Oil transformer cooling radiators should always be equally heat-laden. If one rib, or several of them, is cooler than the other, there is an air pocket inside the radiator or there is not enough oil in the transformer, depending on the load of the transformer and the 
ambient temperature, shown in Fig. 1. The extent of the disorder can vary at certain time intervals, where the balance of physical electrical components can become disturbed, and the impact of the component concerned on the overall security of the system can be reduced.

Risk assessment and model of action are not based on one or two images of the thermal state, but are instead based on continuous thermal images monitoring of the components of the affected LV (low voltage) network.

\section{Convolution Neural Network}

The architecture of CNN is developed mainly based on the research of the human visual cortex. The CNN design is based on the biological architecture of HVS (the human visual system).

Understanding CNN means understanding the anatomy of the visual cortex. The physical location of

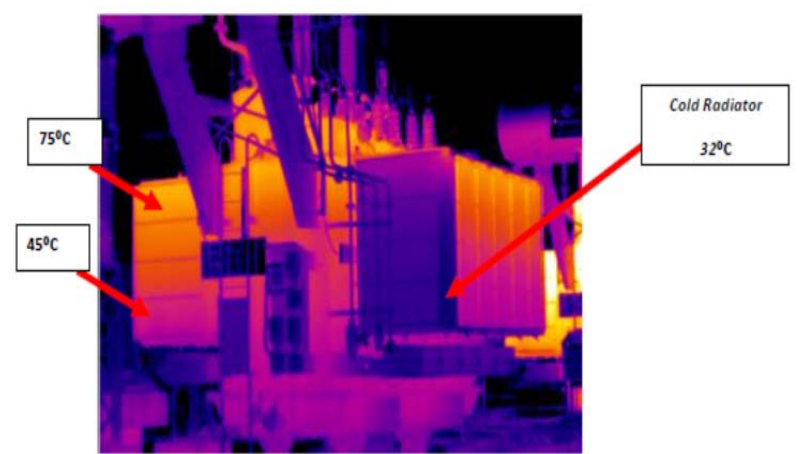

Fig. 1 Thermal image of an oil transformer with oil deficit and air pocket diagnosis.

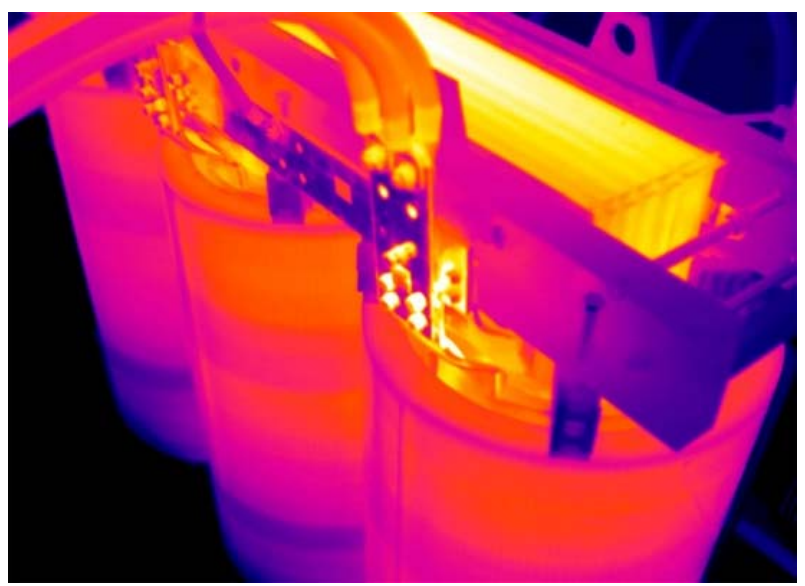

Fig. 2 Thermal image of dry transformer with poor conductor connection. the visual cortex is in the occipital lobe in the back of the head, which has an important role in the processing of visual information received in the brain [2]. The eye as a sensor receives visual information that passes through a number of structures responsible for image processing and they come to a visual cortex.

The part of the brain that receives the input from the sensor (eye) is called the primary visual cortex (V1). The received information is further forwarded through the retinal field, which includes the field of vision V2 and the field of vision V4. Even though other visual fields (V3, V5 and V6) exist, they can not be analysed in this paper because their role is not of interest here. Visual fields V1, V2, and V4, are responsible for shape recognition and are related to the representation of complex parts of objects in an image, for example the face. Fig. 3 shows the ventricular system of the visual cortex.

The ocular retina transforms light energy into chemical energy, which is then in the eye transformed into an electric pulse and transmitted to the visual cortex.

The primary visual cortex V1 has the task of recognizing the boundaries of objects where the edges have a strong contrast in the visual signal, and this clearly shows the transition of one object to another. The first part of the visual cortex associated with associating objects with the terms from memory is V2 (secondary visual cortex). There is a strong connection

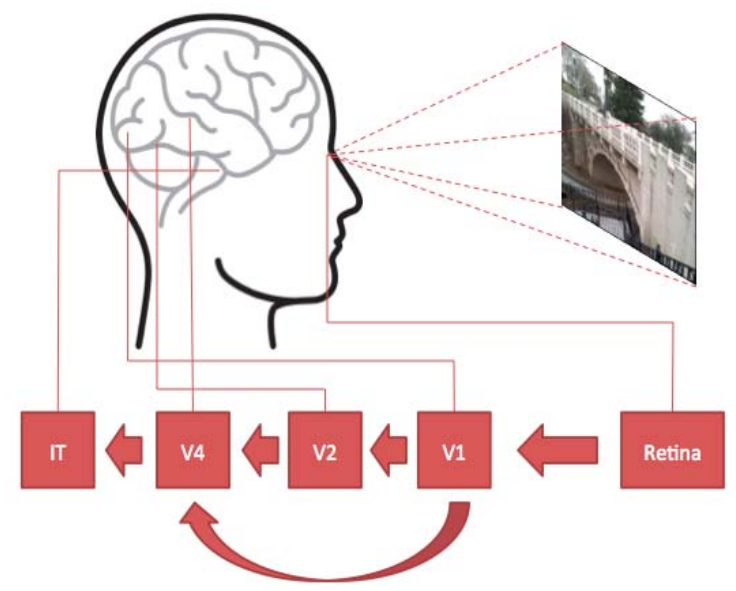

Fig. 3 Ventral visual cortical system in human brain. 
between V2 and V1 that transmits the signal through strong connections to other parts of the visual cortex. In addition, signals related to orientation, spatial frequency, and colour are processed by V2. Part V4 indicates objects related to objects of moderate complexity, such as simple geometric shapes, orientation additions, spatial frequencies or scene scenes. Inferior temporal gyrus (TI) identifies objects in terms of colour and shapes, based on processed information in the object memory. TI performs semantic tasks like facial recognition. With this mentioned part of visual cortex, it is the end of defining CNN through the visual cortex of the brain. The above-mentioned parts of the visual cortex are explained in the context of $\mathrm{CNN}$ for users exposed to technical terms. Based on the above-mentioned parts, CNN comprises: an aggregate layer and layer under the sample. However, the nature of CNN operations is very precise consisting of two mathematical functions (f and $\mathrm{g})$ resulting in a third function $(\mathrm{h})$, which is an integral amount of function f moved from the second function $\mathrm{g}$; the formula is:

$$
h(t)=\int_{-\infty}^{\infty} f(\tau) g(t-\tau) d \tau
$$

Marked as $\mathrm{h}=\mathrm{f} * \mathrm{~g}$.

Most of the time, CNN operates with a 2-D set operations presented in Fig. 4.

The left matrix in Fig. 4 is an input matrix, while the middle square is called a "grain" matrix. These matrices are subject to aggregate operation resulting in the matrix that is on the right side of the image. The process of generating the aggregate operation is performed on the elements and then followed by the sum of the elements in the new signal. In this example, can be seen that when the upper left-hand side (colour) of the input matrix $(3 \times 3)$ is "wrapped" with the grain matrix, the result equals 29 . When we move one field to the right and re-segment the $3 \times 3$, the convolvulus grain matrix the resulting figure is 12 . We apply the same principle to create an output matrix. As the "grain" matrix is $3 \times 3$, any target matrix must also be $3 \times 3$ and consequently the resultant matrix. If we take a $5 \times 5$, after a series of operations on that matrix, it will be reduced to a $3 \times 3$ matrix. This means that the any schedule that will be in the $3 \times 3$ matrix in the $5 \times 5$ input sequence, will result in 29.

This method for disregarding a schedule is of essential for the problems related to visual situations that require recognition effort. In a concrete situation, the rotation or orientation of an object shape in the image or parts of it should render its recognition unfeasible. In $\mathrm{CNN}$, the matrix series process elegantly resolves this barrier. In order to link the visual maps and the aggregate operation, we have to presume that the input matrix of $3 \times 3$ images has to be processed. Fig. 5 shows an analogue visual cortex and an aggregate matrix with an input matrix.

As can be inferred, different series enable various analyses to be applied to the same picture. This allows a successful analysis, and it is advisable to combine several grain textures in one and the same image. Fig. 5, under (d) depicts grain texture that isolates the sharp edges of objects in the picture, which is one of the roles the visual cortex in the human brain, although CNN has an alternative in the form of RNN (Recurrent Neural Network) [11]. RNN is a deep neural network architecture for object recognition based on the modification of replacing the ubiquitous convolution in combination with pooling layer of the deep CNN with four RNN that sweep horizontally and vertically in both directions across the image. The tool for RNN is called ReNet, which is alternative to AlexNet, mentioned below.

Artificial Neural Network (ANN) is based on natural neural network architecture present in brain. Based on

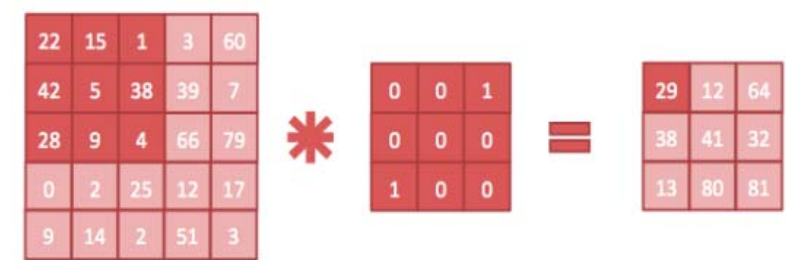

Fig. 4 A simplified presentation of a 2-D group operation based on Eq. (1). 


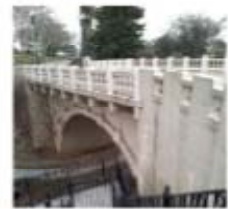

米
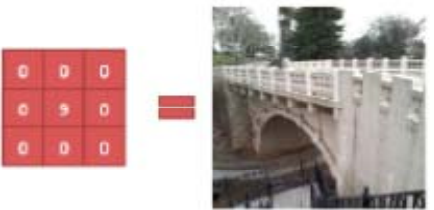

(a) Identification grain

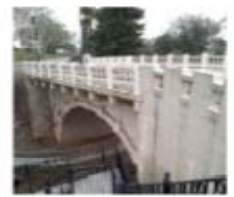

inc

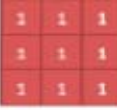

(c) Blur grain

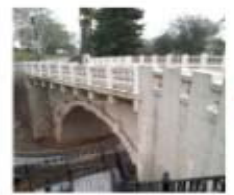

幽

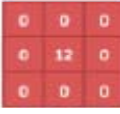

(e) Light grain
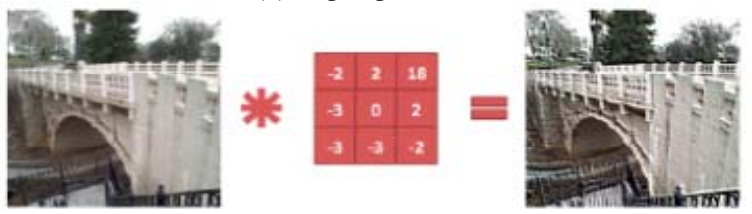

(g) Custom grain 1
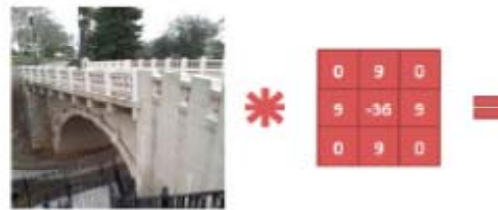

(b) Border grain
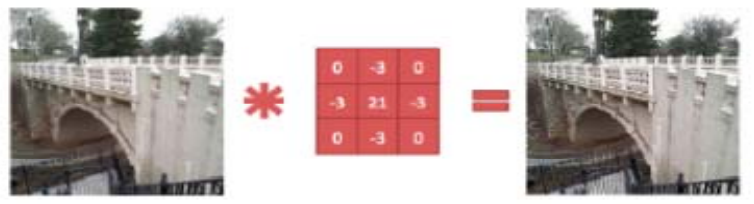

(d) Sharpe grain
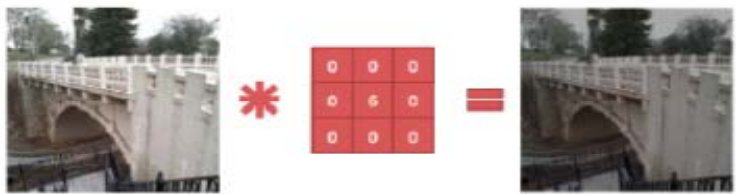

(f) Dark grain
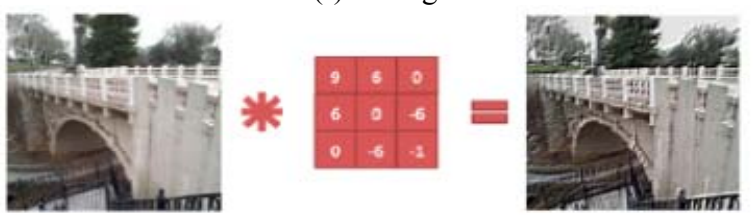

(h) Custom grain 2

Fig. 5 The overall operation of the picture. Different grains added to the same image will result in different processed images. Coloured matrix is "grain" matrix.

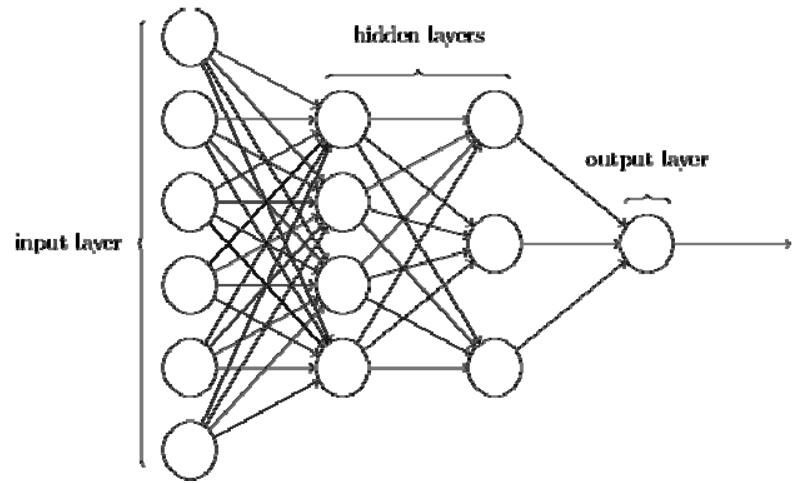

Fig. 6 Basic structure of ANN based on brain neuron network.

natural architecture of neurons ANN comprises 3 three basic elements: an input layer, a hidden layer and an output layer, as presented in Fig. 6. As name says input layer is first to be activated with input variables converting them to values suited for hidden layer. Hidden does not mean "not visible" for user, it just stays for "not input" and "not output". In Fig. 6 it is presented ANN with 2 hidden layers. There are other structures of ANN not necessary same, some of them have feedback loop connection to input layer so training is done more accurately. Those NNs are called Recurrent Neural Networks. Neurons outputs are calculated based on their function, input and connection weight.

$$
a_{j}(t+1)=f\left(a_{j}(t), p_{j}(t), \theta_{j}\right)
$$

where,

$f$ is activation function for computing new activation, $a_{\mathrm{j}}$ is activation;

$\theta_{\mathrm{j}}$ is threshold for $\mathrm{j}$ neuron;

$p_{\mathrm{j}}$ is input from predecessor neuron.

Eq. (1) is computed for every neuron giving at the output new value as input for next layer neuron.

\section{Case Sample}

The simulation of a DL goal locating fault is carried out using a 10/0.4 kV transformer in a distribution substation. The main goal is locating heat dissipation. The DL algorithm is able to send alert or/and report to the user about cabinet condition, depending on the 
speed of heat dissipation, high spot temperature and location. In this way any greater damage could be avoided, in case that the heat is high enough to induce evaporation of oil or losses in power. Besides that, possible damage could be avoided when the temperature is rising with the constant current. User needs to provide IR images of cabinet regardless of the temperature of the cabinet (fault or not fault). In this way, the difference of temperature will be easily spotted by DL. The other possible way is to provide images only without fault but, in that case, the user has to train it through $\mathrm{CNN}$ to report on any difference in images in the future. All images are taken with a thermal (IR) camera.

In order for Matlab to work with photos uploaded by a user, a trained CNN add-in should be introduced that can differentiate the detailed elements in images. This network can be downloaded as a Matlab add-on to the working environment within Matlab. For the development of CNN environment, we are depending on "Alexnet", which is the result of training of thousands of different images to help the CNN network recognize the object when it is introduced an input image. The part of the analysis required for this example takes place in one of the channels for image analysis, which focuses only on that part of the image which the user wants. First, we take the CNN network and instantiate it in another variable. The command is the following:

$$
\text { net }=\text { alexnet; }
$$

The network is loaded and it shows on the workspace. The image is automatically stored in the Matlab workspace. Fig. 7 shows an uploaded IR image in workspace that is ready for processing.

A list of available layers that we can use it with our picture is issued, and a listing of functions is needed need to this end. The aggregate layer algorithm uses learned parameters. The network itself learns how to use features, and it is common one feature per layer.

The network can train using much larger dimensioned images, but this requires a lot of processor time and operating memory. The next step explores the features of the area in the activity degree relative to the original image. Active hot spots are searched and the transformer's focal points are located. On the basis of the number and intensity of these points, it is concluded whether or not the transformer is in the normal mode of operation, so the following triggered activity raises the alarm or activates the protection switch. Each layer of CNN consists of several 2D rows called "channels".

To see the images of the entire network and view all outgoing output activation slogans, we include "conv1".

Activations return as $3 \mathrm{D}$ rows, with a third dimension indexed on the "conv1" layer. To see these activations using the "montage" function, it is needed to change the order in $4 \mathrm{D}$. The third dimension in the "montage" input represents the colour of the image. Set the third dimension to 1 , so the colour will be omitted because the intensity of the light on the picture does not require colour, as in Fig. 8. The channel index represents the fourth dimension.

Each activation can contain some values. That is the reason the output normalizes if the function "mat2gray" is used. All color-coded activations are scaled with a minimum of 0 and a maximum of 1 . Positive activation is represented by white pixels, while negative activation is represented by black pixels. In most cases, the grey channel is not activated so strongly in the

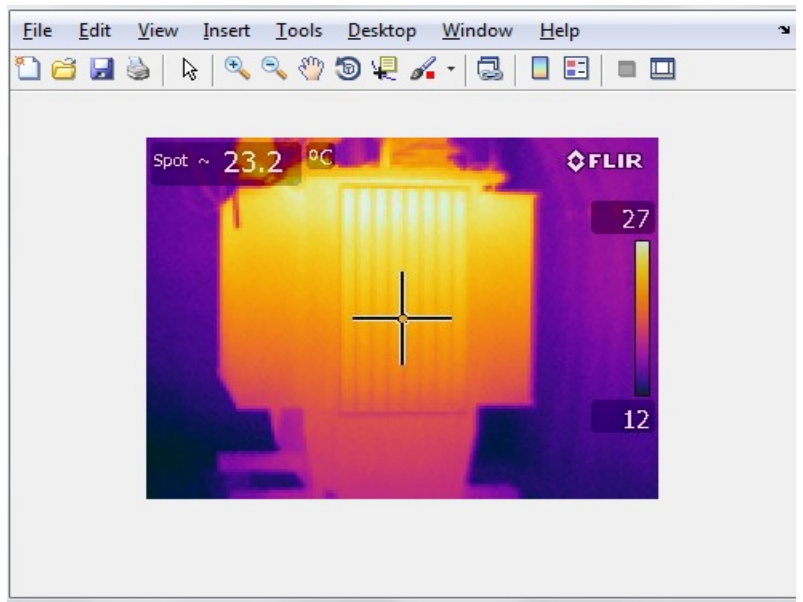

Fig. 7 Original image of transformer IR camera uploaded to Alexnet network. 


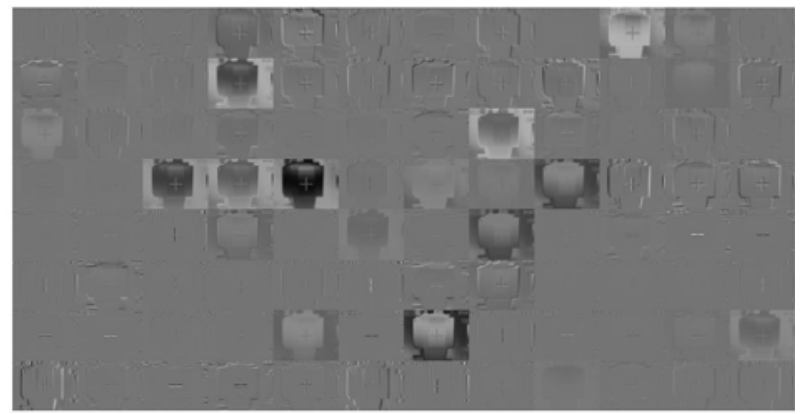

Fig. 8 Channel activation for image analysis with no colour and with light on individual images.

original images. It is very important that the position of pixels on the activation channel corresponds to the same position in the original image. The white pixel on the same spot in the channel shows strong channel activation in the original images. In Fig. 9, the size of channel 32 equals the size of the original image.

This channel is activated in red because the white pixels are in the position in the original picture in the place of red colour, reference in Fig. 10. It is possible to extract the channel with the strongest activation.

CNN learns to notice features, such as colour or shapes, in the image at its first bulk level. At deeper aggregate levels, the network learns to see details that are more complicated by combining the results from previous levels, presented in Fig. 11. For the purpose of this example, examine the "conv5" layer in the same way as "conv1".

Focus on the most active layer.

This channel focuses on the white colour and shapes of the picture that are not perfect for our analysis. With a more detailed analysis of the presented channels, the number 32 focuses on parts of the original image, presented in Fig. 13.

Repeat the analysis to visualize activation of the "relu5" layer. In order to leave only positive activations, the correction unit $(\mathrm{ReLu})$ is used as reference in Fig. 14.

Compared to the activation of the "conv5" layer, activation on the "relu5" layer clearly shows the zones that are thermal centres on the original image. In order to detect a change in heat output, it is essential to compare the current image with a new image from the same angle shot with the same camera at a different time so that the difference in the pixels in the "relu5" layer is accurately visualized.

Final image for clear recognition of focus area is presented in Fig. 15 where visible activation pixels are. User without scripted sequence does all above process. Current example presents normal transformer operational mode with no fault present.

Presented image can be used in programmed environment for calculating area, which is critical for

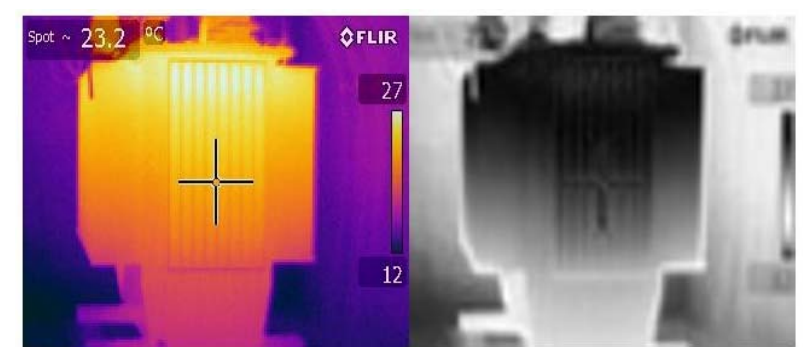

Fig. 9 Channel 32 in size as the original image and original image left.

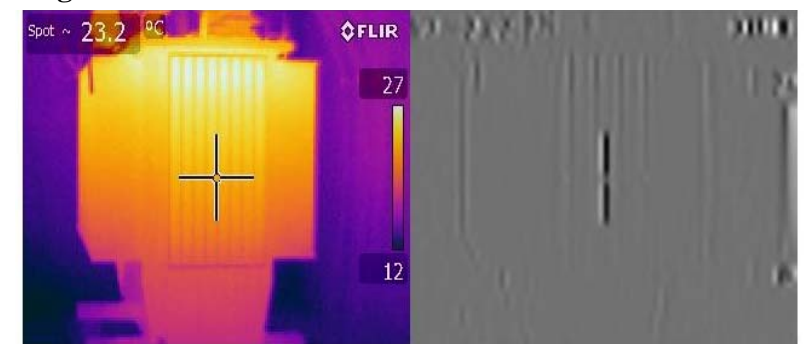

Fig. 10 The strongest activation channel. The channel changes to the black and white transition on the original image.

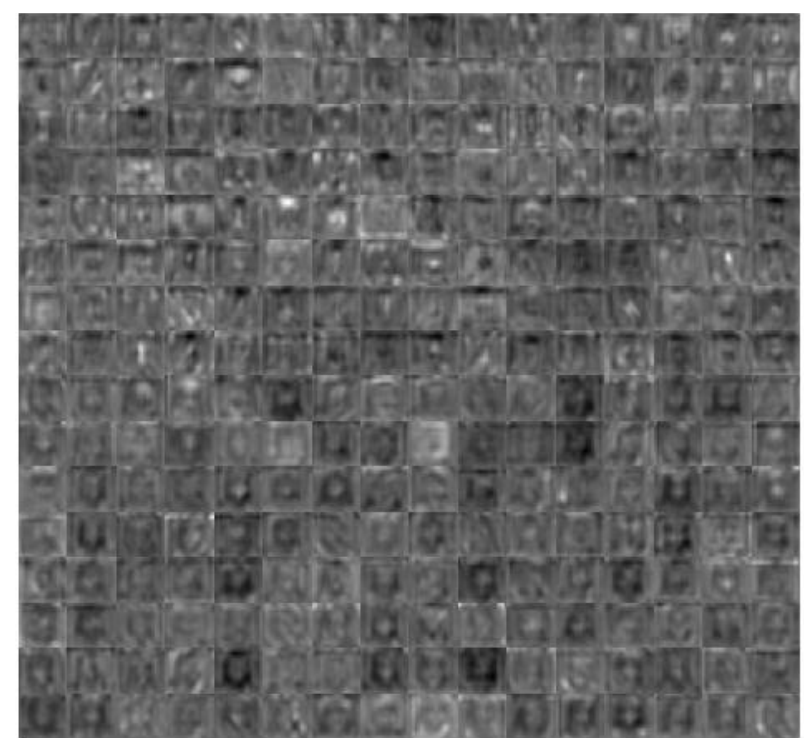

Fig. 11 Conv5 channel presents layers suitable for analysis. 


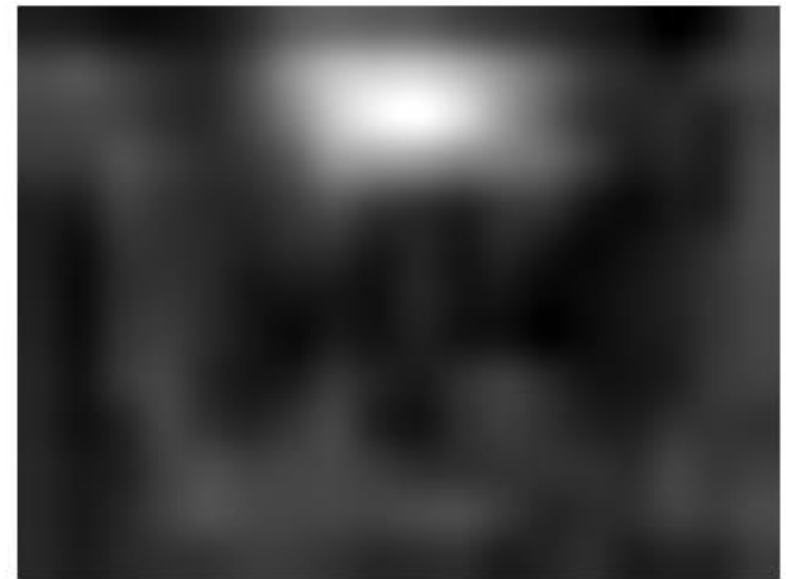

Fig. 12 Most active channel on conv5 layer regarding original image.

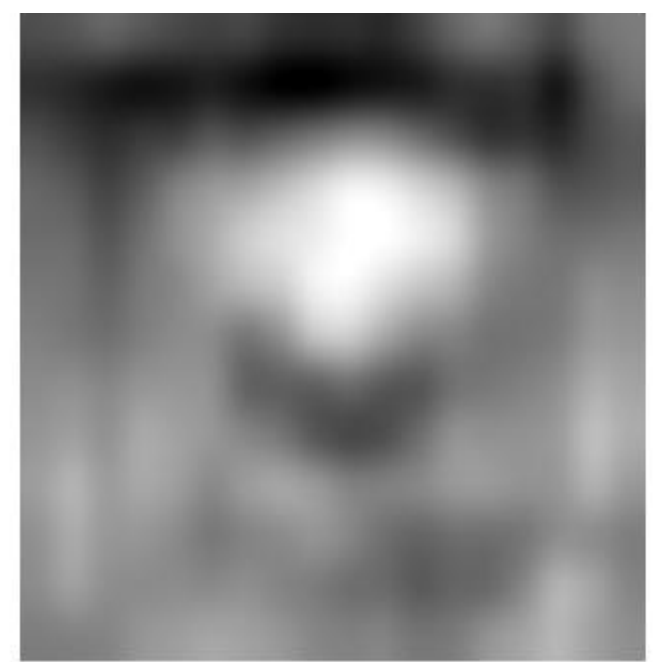

Fig. 13 Channel 32 with focus on the bright parts of the original image.

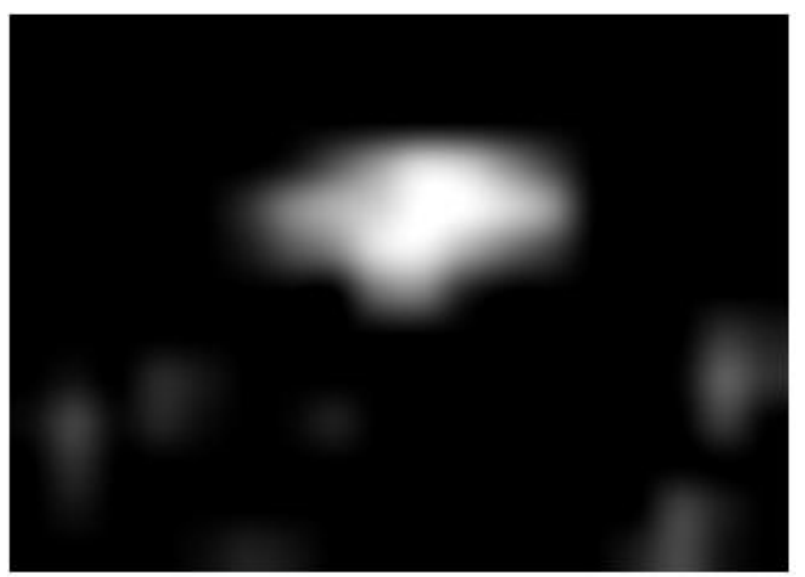

Fig. 14 ReLu activated on conv5 layerand obvious focal point.

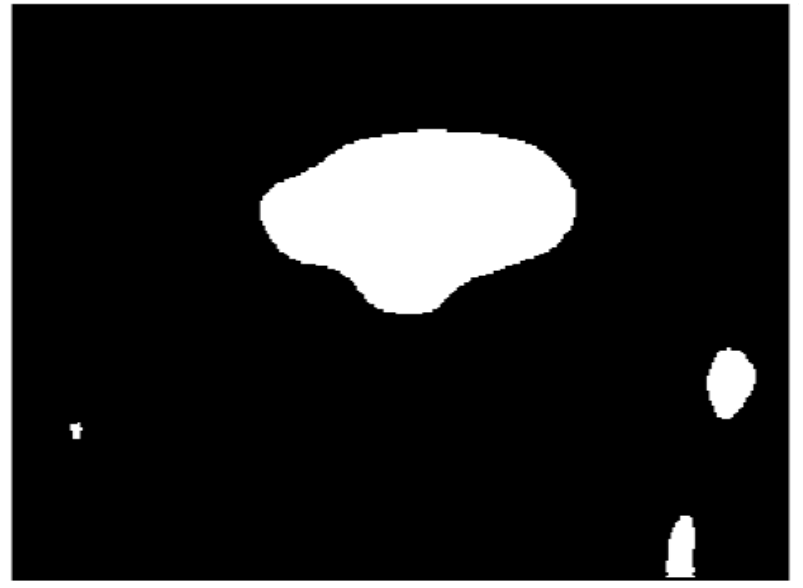

Fig. 15 Final image for clear recognition of focus area.

fault warning or even protection measures sequence activation.

The script can be placed in an uninterrupted loop and saved as such. When needed, the script is started and the images are taken in real time from the camera and analysed. The script will analyse all previous images and thus make a conclusion about the state of the transformer, the impact intensity, the temperature and, if necessary, the heat energy emitted and thus easily get the energy losses in the power grid. On the basis of this information, it is possible to take timely action and prevent failure of the distribution network, or potential fire, etc.

\section{Conclusion}

This work presents the AI Deep Learning method with a CNN network trained and tested for recognizing transformer images, and the considered method is presented through a sample case of a 10/0.4 $\mathrm{kV}$ distribution network transformer. The use of AlexNet tool is explained in the paper. The method is used to analyse the faults detection in the transformers in the distribution network. Section 3 of the paper demonstrates that the $\mathrm{CNN}$ network is flexible, meaning it is appropriate for situations requiring power system expert analysis. Through the carefully trained 
NN network with carefully selected impulses, algorithms with operations and matrices skilfully simulate human inference mechanism and thereby reduce the resources needed for the process of analysis. The ultimate result is a timely action in terms of protecting people and equipment. Further research on this method will compare results against other tools, like the above-mentioned ReNet.

\section{References}

[1] Bradley Erickson, Panagiotis Korfiatis, Zeynettin Akkus, and Kenneth Philbrick. 2017. "Toolkits and Libraries for Deep Learning.” Journal of Digital Imaging, March 2017, Article, DOI: 10.1007/s10278-017-9965-6.

[2] Hinton, G. E., Osindero, S., and The, Y-W. 2006. "A Fast Learning Algorithm for Deep Belief Nets." Neural Comput. 18: 1527-54.

[3] Krizhevsky, A., Sutskever, I., and Hinton, G. E. 2012. "Imagenet Classification with Deep Convolutional Neural Networks." In: Advances in Neural Information Processing Systems 25. Pereira, F., Burges, C., Bottou, L., Weinberger, K. Q. editors.

[4] Wang, H. H., and Bhiksha, R. 2017. "On the Origin of Deep Learning."

[5] Keiichi Osako, Rita Singh, and Bhiksha Raj. 2015. "Complex Recurrent Neural Networks for Denoising Speech Signals.” 2015 IEEE Workshop on Applications of Signal Processing to Audio and Acoustics (WASPAA),
October 2015, DOI: 10.1109/WASPAA.2015.7336896.

[6] Aman Gupta, Haohan Wang, and Madhavi Ganapathiraju. "Learning Structure in Gene Expression Data Using Deep Architectures, with an Application to Gene Clustering." 2015 IEEE International Conference on Bioinformatics and Biomedicine (BIBM), November 2015 DOI: 10.1109/BIBM.2015.7359871.

[7] Leon, A., Gatys, Alexander S Ecker, and Matthias Bethge. 2015. “A Neural Algorithm of Artistic Style.” Journal of Vision, August 2015, DOI: 10.1167/16.12.326.

[8] Jehan Shazly, Doaa Ibrahim, Essam Abo El Zahab, and Mohamed Mostafa. 2017. "Thermal Analysis of HV Cables with Several Types of Insulation for Different Configurations in the Presence of Harmonics." IET Generation Transmission \& Distribution, June 2017, DOI: 10.1049/iet-gtd.2016.0862

[9] Suwarno Harjo, and Rizky Auglius Pasaribu. 2016. "Thermal Aging of Mineral Oil-Paper Composite Insulation for High Voltage Transformer.” International Journal on Electrical Engineering and Informatics 8 (4): 820-35, December 2016, DOI: 10.15676/ijeei.2016.8.4.9

[10] American Society for Non-destructive Testing. 2011. "ASNT Standard for Qualification and Certification of Non-destructive Testing Personnel (ANSI/ASNT CP-189-2011) \& ASNT Std." Topical Outlines for Qualification of NDT Personnel (ANSI/ASNT CP-105-2011).

[11] Francesco Visin, Kyle Kastner, Kyunghyun Cho, Matteo Matteucci, Aaron Courville, and Yoshua Bengio. 2015. "ReNet: A Recurrent Neural Network Based Alternative to Convolutional Networks." Arxiv:1505.00393. 